\title{
Modeling of A LDR Based Liquid Level Detecting Device Using Power Function
}

\author{
Raifu Ishola SALAWU ${ }^{1}$, Sunday Oladejo ADETONA ${ }^{2}$ \\ ${ }^{1}$ Department of Electrical \& Electronics Engineering, Bells University of Technology, Ota, Nigeria \\ ${ }^{2}$ Department of Electrical \& Electronics Engineering, University of Lagos, Lagos, Nigeria \\ *e-mail: sadetona@unilag.edu.ng
}

Received: $29^{\text {th }}$ April 2021

Accepted: $13^{\text {th }}$ May 2021

Published: $5^{\text {th }}$ August 2021

https://doi.org/10.47545/etrj.2021.6.2.080

\begin{abstract}
All vehicles require liquid to move from one point to the other; and it is necessary for the driver of the vehicle to ascertain the volume (level) of the liquid in the tank before setting out on a journey, hence the need to know the level of the liquid in the tank. This paper therefore presents modeling and simulation of a sensory device, which evaluates the volume $\mathrm{V}_{\mathrm{LQD}}$ of any type of liquid in any type of a closed container that has a cross-sectional area A $\left(\mathrm{m}^{2}\right)$ and height $\mathrm{h}(\mathrm{m})$. The main attraction of the approach is that it does not get in touch with the liquid, its simplicity and lower cost. The aim is achieved by using a sensor, Light Dependent Resistor (LDR); which operates on a principle which states that the voltage $E_{\text {LUX }}$ in Volts across a LDR is a function of luminance it receives from a light source, Light Emitting Diode (LED). This principle and a power function developed using experimental data were used to model $V_{\mathrm{LQD}}$; which reveals that $\mathrm{V}_{\mathrm{LQD}}$ in litres is a function of $\mathrm{E}_{\mathrm{LUX}}$ in Volts. The model of $\mathrm{V}_{\mathrm{LQD}}$ was simulated for the sake of validation in the Proteus 8.9 professional environment. The results obtained revealed that the proposed model correctly indicated the $\mathrm{V}_{\mathrm{LQD}}$ in litres in a closed container that its $\mathrm{A}\left(\mathrm{m}^{2}\right)$ and $\mathrm{h}(\mathrm{m})$ are known and specified in the model.
\end{abstract}

Keywords: Inverse Square law, Light Dependent Resistor, Liquid, Power function, Sensor

\section{INTRODUCTION}

There are various methods of detecting liquid level in a container. One of these approaches is the one proposed by [1]; which uses the principle of buoyancy that states that a float immersed in a liquid is buoyed in an upwards direction by an applied force equal to the weight of the displaced liquid. As a result, the body moves partially and gets submerged on the liquid surface and covers the same distance that the liquid level moves. The studies in [2], [3], and [4] employed capacitance effects in the development of their various forms of liquid level detectors; which are based on the change of capacitance of the sensor. In this sensor, there are two plates, one of which acts as an insulated electrode; and the other plate acts as the wall of the tank. The value of the capacitance depends upon the level of the liquid in the tank. When the tank is empty, it acts as a low capacitance and when full, it acts as a high capacitance. Another set of liquid level detector are those proposed by [5], [6] and [7]; which are based on the conductivity of probes. In this approach, two electrodes are placed in the liquid and the conductivity of the liquid depends upon the level of the liquid in the container. The liquid detector proposed by [8], [9], and [10] makes used of the ultrasonic sensor; which is made of two units, consisting of a transceiver and a transducer. The liquid level is determined by measuring the trip time difference between a transmitted ultrasonic pulse and its reflected echo. These sensors have the advantage that they are not in direct contact with the liquid and the level measurement is achieved without any physical contact with the liquid whereas, in [9] and [10] liquid level detection uses radar. The principle of radar level detector is similar to that of the ultrasonic sensor; that is, measuring the time require for a microwave pulse and its reflected echo to make a complete return trip between the non-contacting transducer and the liquid level. The transceiver then converts the travel time to distance and presents it as the level of the liquid. Of all level detectors discussed above, only the radar and ultrasonic sensors do not get in contact with the liquid whose height is being measured; hence they do not introduce dirt into the tank. As in the cases of Radar and Ultrasonic detectors, the proposed approach does not get into contact with the liquid, but has the additional advantage of simplicity and low cost.

The principle of the proposed approach is similar to that of the ultrasonic and radar sensors in that it does not get into contact with the liquid, but dissimilar in that it does not measure the time require for the illumination of the light and its reflection in a complete return trip between the non-contacting Light Dependent Resistor (LDR) and the liquid level. In this contribution, the quantity of lux received by the LDR causes the resistance of the LDR to 
change with the level of the liquid in the closed container, and this generates voltage across LDR, which is directly related to the distance between the top surface of the liquid and inside cover of a closed container.

\section{METHODOLOGY}

\subsection{Design Analysis and Considerations}

Figure 1 presents the physical model of the detector placed inside cover of a closed container.

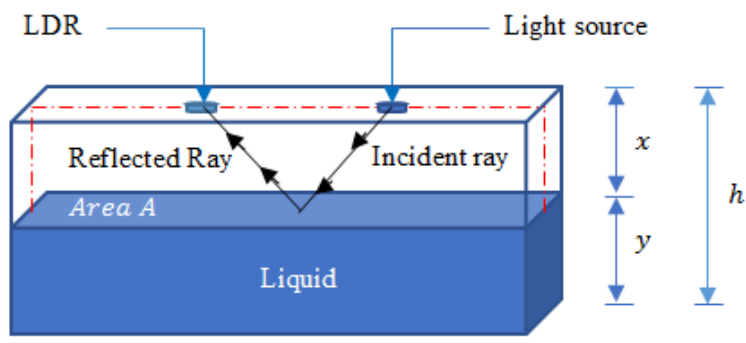

Figure 1. The physical model of the proposed device

If it is assumed that the total height of the closed container is $h(m)$ and the distance between the top surface of the liquid and the inside cover of the closed container where the LDR is mounted is $x(m)$, then the height of the liquid $y(m)$ in the container is therefore

$y=(h-x)$

If the cross-sectional, area of the closed container is $A\left(\mathrm{~m}^{2}\right)$, then, the volume of the liquid, $V_{L Q D}$ (litres), in the container is

$V_{L Q D}=1000 A(h-x)$

Thus, as $x$, which is a variable of interest changes, the volume of the liquid in a closed container also changes. In this contribution, the LDR shown in Figure 1 is used to detect $x$ in a closed container. The one used is the cadmium sulfide $(\mathrm{CdS})$ photocell. It has a peak spectral response around the wavelength of about $610 \mathrm{~nm}$ in the red to orange region of light. When in the dark, it has a resistance that is very high (of the order of about $10 \mathrm{M} \Omega$ ); which varies from that value to about $1 \mathrm{k} \Omega$ when fully illuminated. The CdS photocell is shown with its substrate and symbol in Figure 2; and its characteristic is presented in Figure 3. Modern LDRs are made of lead sulfide, lead selenide, indium antimonide, and most commonly, CdS. The characteristics of CdS reveal that a non-linear decrease in resistance when illuminated, that is, the higher the illumination, the lower the resistance $\mathrm{R}_{\mathrm{LUX}}$ of the LDR.
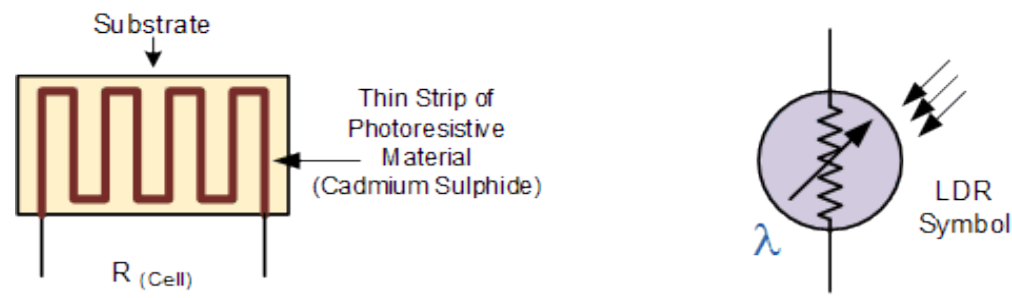

Figure 2. The substrate and symbol of the CdS photocell

Figure 4 shows the schematic diagram of the LDR; which reveals that when it is biased and energized, the voltage $E_{L U X}$ will drop across its resistance $R_{L U X}$. The quantity of lux received by the LDR determines $E_{L U X}$, which is a function of $R_{L U X}$, bias resistor $R_{B}$, and the source voltage, $V_{C C}$ and can be expressed by using voltage divider rule

$E_{L U X}=\frac{R_{L U X}}{R_{L U X}+R_{B}} \times V_{C C}$

But $R_{L U X}$ is a function of the luminous intensity $I$ received by LDR, as shown in Figure 1 and revealed by [11] to be 
$R_{L U X}=\frac{R_{0}}{1+\alpha I^{\beta}}$

where $R_{0}$ is LDR dark resistance, $\alpha$ and $\beta$ are constants [11] and their values depend on the colour of the light received by the LDR. In Equation (4), $I$ is measured in $\mu W / \mathrm{cm}^{2}$ [11]; therefore, the equation can be recast as

$R_{L U X}=\frac{R_{0}}{1+\alpha\left(10^{-6} I\right)^{\beta}}$
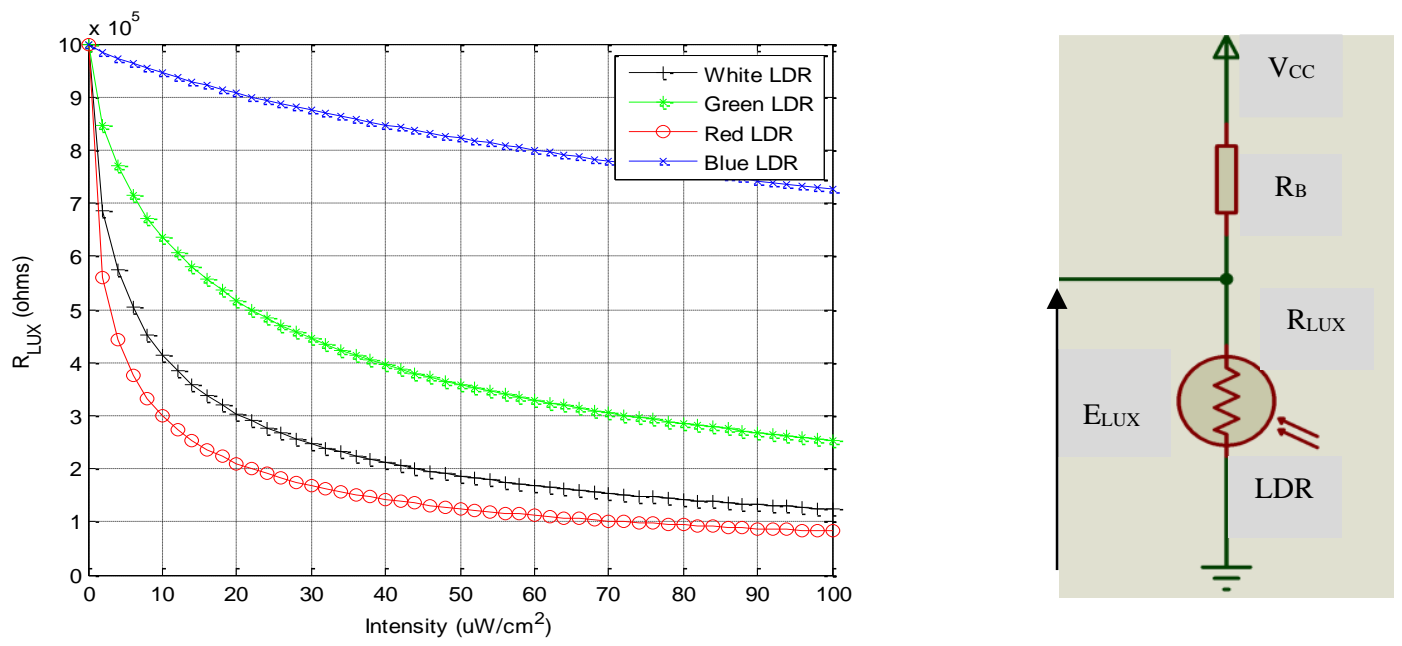

Figure 3. The characteristic of the CdS photocell [11]

Figure 4. The schematic diagram of the CdS photocell

Upon using Equation (5) in Equation (3), we have;

$E_{L U X}=\frac{R_{0}}{R_{0}+R_{B}\left(1+\alpha\left(10^{-6} I\right)^{\beta}\right)} \times V_{C C}$

The plot of $E_{L U X}$ against $I$ using parameters provided in [11] for different colours of light received by LDRs is presented in Figure 5; which reveals that the quantity of lux received by the LDR determines $E_{L U X}$.

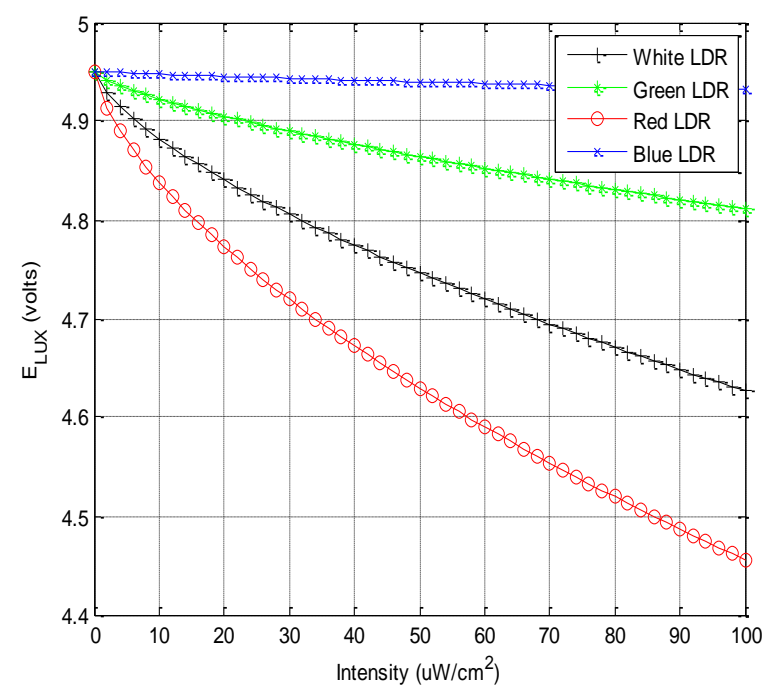

Figure 5 The plot of $E_{L U X}$ against $I$ using parameters for different colours of light received by the LDR

And upon expressing $I$ in Equation (6) as the subject of the formula we have;

$I=\left\{\frac{1}{\alpha}\left[\frac{R_{0}}{R_{B}}\left(\frac{V_{C C}}{E_{L U X}}-1\right)-1\right]\right\}^{\frac{1}{\beta}} \times 10^{6}$

It can be established from Equation (2) that $V_{L Q D}=f(x)$; and, $I$ must therefore be linked to $x$. One of the ways of achieving this is by invoking Inverse Square Law (ISL), which states that the luminance is proportional to the inverse square of the source-sensor distance [12]; that is 
$I=\frac{k}{x^{2}}$

In Equation (8), $k$ is a proportionality constant. It should be pointed out here that Equation (8) is a customized version of Power equation; which is better expressed as:

$I=k x^{n}$

In term of the ISL, $n=-2$. It is evident from Equation (9) that $k$ and $n$ are unknown constants; and their values can only be obtained through experimental evaluation. In this contribution, in order to obtain an expression for $x$, Equations (7) and (9) are equated, from which, $x$ is expressed as

$x=\left(k \alpha^{\frac{1}{\beta}}\right)^{-\frac{1}{n}}\left\{\left[\frac{R_{0}}{R_{B}}\left(\frac{V_{C C}}{E_{L U X}}-1\right)-1\right]\right\}^{\frac{1}{n \beta}} \times 10^{-5}$

The distance between the top surface of the liquid and the inside cover of the closed container where the LDR is mounted is given in Equation (10); hence, the volume of the liquid in the closed container is

$V_{L Q D}=1000 A\left(h-\left(\left(k \alpha^{\frac{1}{\beta}}\right)^{-\frac{1}{n}}\left\{\left[\frac{R_{0}}{R_{B}}\left(\frac{V_{C C}}{E_{L U X}}-1\right)-1\right]\right\}^{\frac{1}{n \beta}} \times 10^{-5}\right)\right)$

In Equation (11), the following parameters: $k, n, R_{0}, \alpha, R_{B}, V_{C C}$, and, $\beta$ are all constants; therefore, it can be stated that the volume (litre) of any type of the liquid in a closed container of area $A\left(m^{2}\right)$, and height $h(m)$ is just a function of the $E_{L U X}$ across LDR only.

\subsection{Realization of parameters for the proposed device}

Determination of values of $\boldsymbol{R}_{\mathbf{0}}, \boldsymbol{\alpha}$ and $\boldsymbol{\beta}$ : According to [12] the values of $\boldsymbol{R}_{\mathbf{0}}, \boldsymbol{\alpha}, \boldsymbol{a n d} \boldsymbol{\beta}$ in Equations (10) and (11) are function of the colour of light received by the LDR used. In this paper, red LED was used as the light source, because red beam of light can be dispersed easily when reflected from the surface of the liquid [12]. The values of $\boldsymbol{R}_{\mathbf{0}}, \boldsymbol{\alpha}$, and $\boldsymbol{\beta}$ based on red colour received by $L D R$ are $\mathbf{1 0 0} \times \mathbf{1 0}^{\mathbf{6}} \boldsymbol{\Omega}, \mathbf{5 8 8 5}$, and 0.68 [11] respectively.

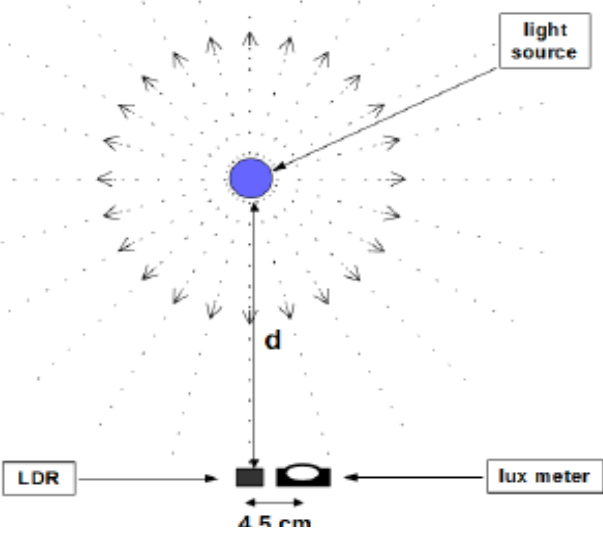

Figure 6 Experimental setup of measuring light with LDR [12]

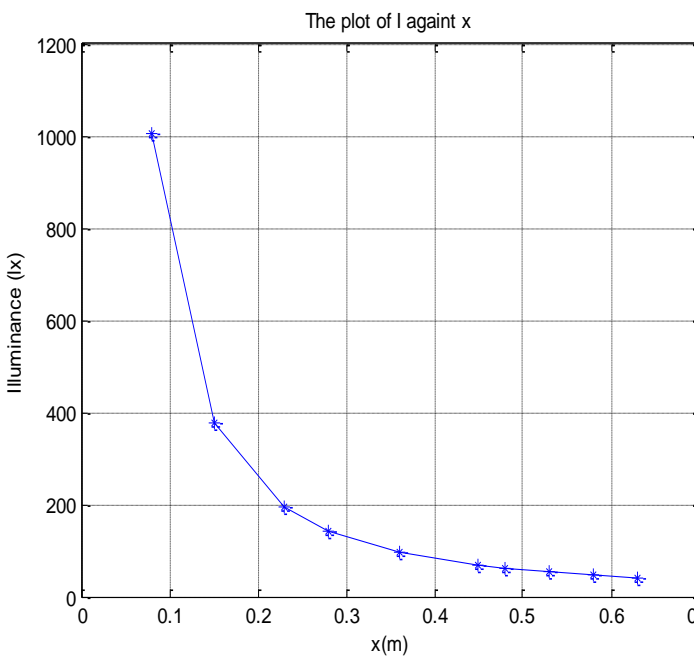

Figure 7 The plot of $I(l x)$ against $x(m)$

Determination the values of $\boldsymbol{k}$ and $\boldsymbol{n}$ : For the purpose of computing the values of $\boldsymbol{k}$ and $\boldsymbol{n}$ in Equations (10) and (11), the experimental procedure in measuring light with LDR proposed by [12] was adopted and implemented.

The representation of the experimental setup is presented in Figure 6. The components used were Red colored LED as a light source, CdS LDR as a sensor, and a lux meter. The distances from the light source to the CdS LDR were varied and recorded; and illuminances on the lux light meter were read and registered. The data are presented in graphical format in Figure 7. The equation that best describes the curve depicted in the figure is a power equation [13]; which is 
In order to find the constants $k$ and $n$, Equation (12) is linearized [13] viz:

$\log _{10} I=n \log _{10} x+\log _{10} k$

The equation obtained after subjecting the experimental linearized data to polyfit [14][15] command in the MATLAB environment is

$I=19.6517 x^{-1.5576}=\frac{19.6517}{x^{1.5576}}$

The model obtained in Equation (14) is a power function; and it has the value of $k=19.6517 \mathrm{lxm}^{2}$, and $n=$ -1.5576 .

Selection of $\boldsymbol{V}_{\boldsymbol{C} \boldsymbol{C}}$ : In Equation (10), $\boldsymbol{V}_{\boldsymbol{C} \boldsymbol{C}}$ represents the source voltage. For all the components used for the realization of the proposed device, the required voltage ranges from 5-12 V; therefore in this study the lower limit of the source voltage range was taking, therefore $V_{C C}$ is $5 \mathrm{~V}$.

Determination of the value of $\boldsymbol{R}_{\boldsymbol{B}}$ : In order to determine the value of required biasing resistor $\boldsymbol{R}_{\boldsymbol{B}}$ for the realization of the LDR circuit for the proposed device, we recalled Equation (3). Upon working on the equation, we obtained:

$R_{B}=R_{l u x}\left(\frac{V_{C C}}{E_{L U X}}-1\right)$

The $V_{C C}$ is expected to be greater than $E_{L U X}$; hence, in this study, $\frac{V_{C C}}{E_{L U X}}$ is assumed to be about 1.1 ; and the maximum value of the $R_{L U X}$ recorded in darkness during the experimentation is $10 \mathrm{M} \Omega$; therefore, $R_{B}=$ $10 M \Omega \times 0.1=1 M \Omega$.

Upon using all the values of the constants parameters in Equation (10), we obtained

$x=0.2535\left\{\frac{50.0}{E_{L U X}}-11.0\right\}^{-0.9442}$

The distance $(m)$ between the top surface of the liquid and the inside cover of the closed container where the LDR is mounted is given by Equation (16); then the level of the liquid $y(m)$ in the closed container is therefore

$y=h-0.2535\left\{\frac{50.0}{E_{L U X}}-11.0\right\}^{-0.9442}$

As such, the volume of the liquid in litres in the closed container is

$V_{L Q D}=1000 A\left(h-0.2535\left\{\frac{50.0}{E_{L U X}}-11.0\right\}^{-0.9442}\right)$

To determine volume of any type of the liquid in any closed container, Equation (18) can be employed provided the red LDR is employed; and it shows that the volume in litres of any type of the liquid in a closed container of area $A$ in $\mathrm{m}^{2}$, and height $h$ in $m$, is a function of voltage $E_{L U X}$ in volts across LDR.

\subsection{Modeling of the proposed device}

Modeling of the proposed device involves development of hardware and firmware.

\subsubsection{Development of hardware}

The schematic diagram in running mode in Proteus 8.9 professional environment [16] for the realization of the detector is presented in Figure 8.

It has Power Supply Unit (PSU), the Arduino Uno which houses a micro-controller, $20 \times 4$ Liquid Crystal Display (LCD), LDR circuit and a light source. The microcontroller embedded in the Arduino Uno has a processor, memory and peripherals [17][18]; and used in this contribution for real-time control applications with on-chip program memory and devices [17][18]. LCD is used in this contribution to display numerically the volume of any liquid in a closed container in litres. All the units in the proposed device required $5 \mathrm{~V}$ supply; as such, an IC LM7805 voltage regulator was used in the power supply unit. It produces output voltage of $5 \mathrm{~V}$ from $9 \mathrm{~V}$ supply. 


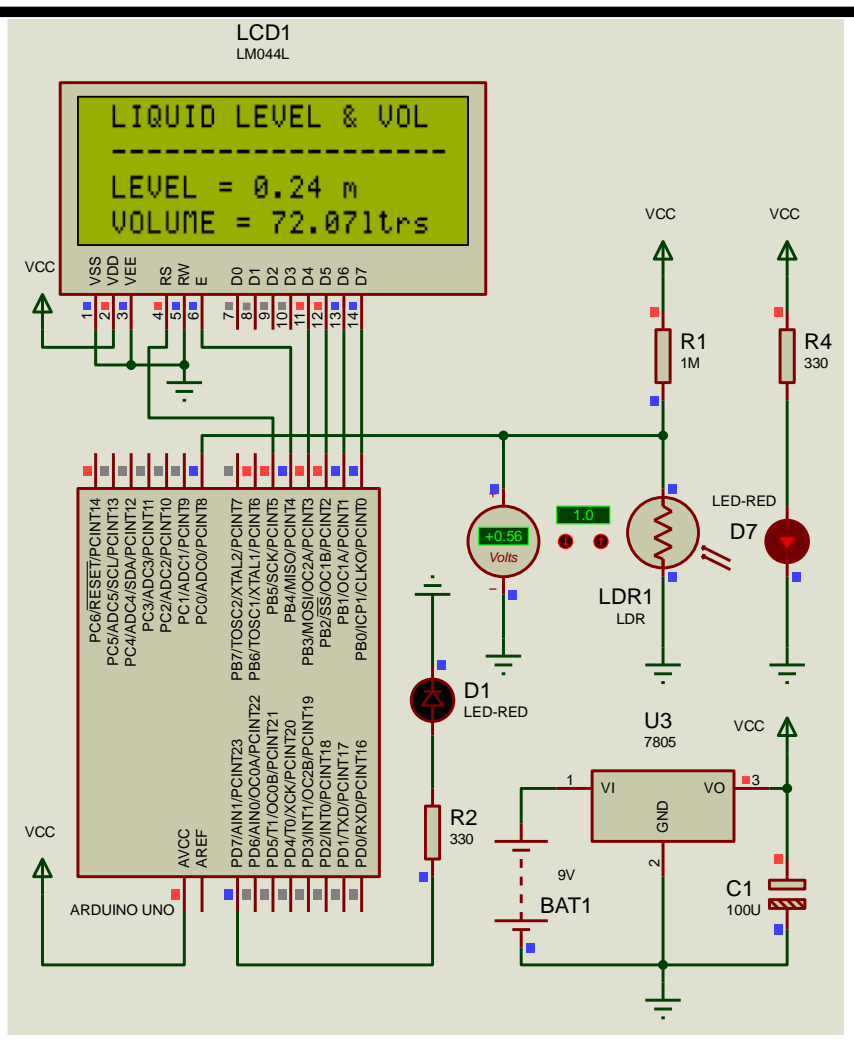

Figure 8 . The schematic diagram of the proposed device

\subsubsection{Development of Software}

Figure 9 shows the flowchart for the development of the firmware for the proposed device. All variables and constants for the computation of level and volume of the liquid in a closed container having area $A\left(\mathrm{~m}^{2}\right)$ and height $h(m)$ are first declared and initialized. These variables are: $P i n A_{0}, E_{L U X}, B, x, A, h$, index, level, and volume. The analog signal that is present at $\operatorname{Pin} A_{0}$ will then be read, and converted to digital signal using the equation and stored the result in variable location named $E_{L U X}$.

The pow $(B$, index $)$ command [19][20] was employed to solve Equation (16); because $C / C^{++}$language that micro-controller that is embedded in the Arduino understands, does not have arithmetic operator for exponentiation [19][20], i.e., $x=B^{\text {index }}$. The value of $x$ obtained is then compared with $h$; which is a constant. If $x$ is equaled to $h$, the LCD displays the level and volume of the liquid in the closed container to be $0 \mathrm{~m}$ and 0 litres respectively; but If $x$ is less than $h$, the level and volume of the liquid in closed container will be evaluated and consequently displayed by LCD. The proposed device stops processing when the level of the liquid gets to $0.27 \mathrm{~m}$, so as to prevent liquid from getting to the positions where LDR and LED are mounted.

\subsubsection{Modeling of the proposed device}

As shown in Figures 1 and 8, the ray of light from the light source is incident on the surface of the liquid; and the ray of light is reflected from the surface of the liquid to the LDR.

The lux of the reflected light on the LDR causes the resistance of the LDR to change with the level of the liquid in the container, and this generates voltage $E_{L U X}$ across LDR. The $E_{L U X}$ is an analog signal which is detected by the micro-controller embedded in the Arduino Uno through pinA 0 of the controller. The micro-controller, which has been programmed, processes the received signal using algorithm presented through flowchart of Figure 9.

\section{RESULTS}

When the schematic diagram which is depicted in Figure 8 was simulated in the Proteus 8.9 professional environment, it indicated correctly the level and volume of the liquid in the closed container that is crosssectional area and height are known. For instance, when the level of the liquid in a closed container that has cross-sectional area of $0.3 \mathrm{~m}^{2}$ and height of $0.3 \mathrm{~m}$ is $0.24 \mathrm{~m}$, its volume was found to be 72.07 litres. This indicates about $0.097 \%$ error. When the illuminations received by the LDR were varied, different levels and volumes of the liquid in the closed container were also indicated on LCD accordingly. 


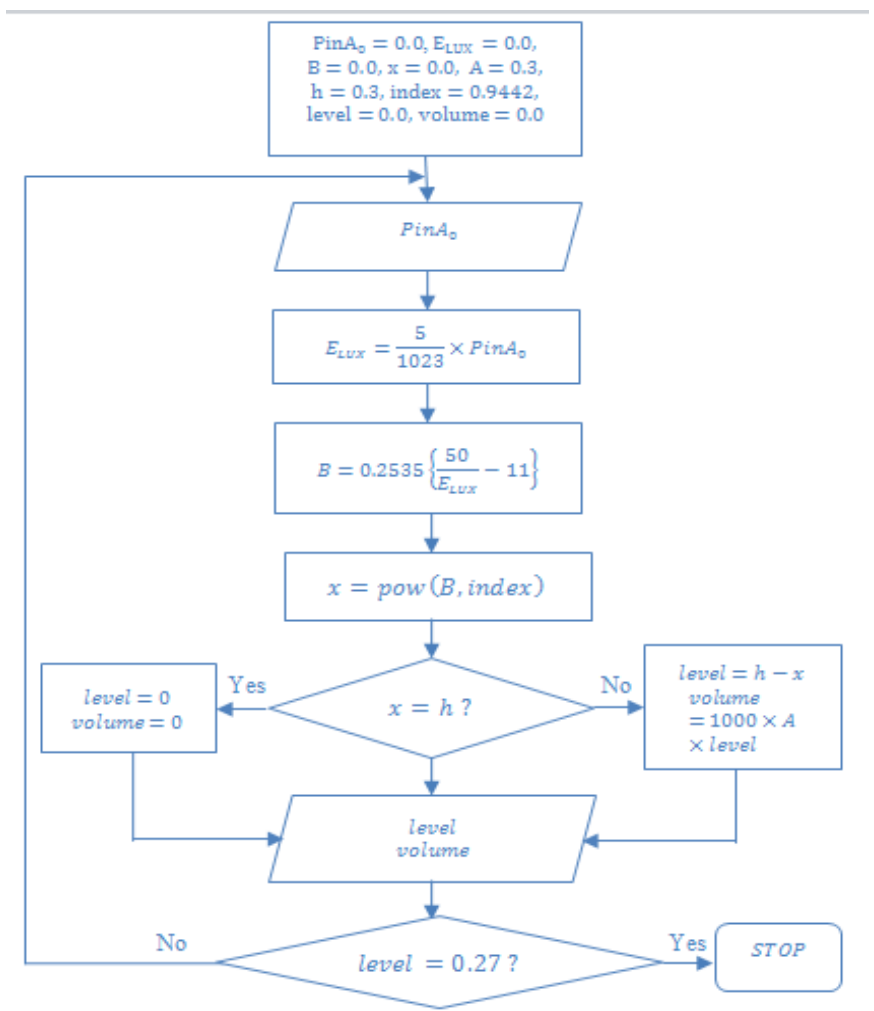

Figure 9 The flowchart for the realization of the firmware for the proposed device

\section{CONCLUSION}

This contribution discusses the application of LDR in determining the height or volume of any liquid in a closed container. The approach does not have any contact with the liquid, therefore there is no likely hood of dirt being introduced in to the liquid in the container, and neither can there be any reaction between the liquid and the ray of light. It uses monochromatic light; because monochromatic light is dispersed when reflected from the surface of a liquid.

The main goal of the study was achieved by using the LDR which operates on the principle that states that the voltage $E_{L U X}$ across an LDR is a function of the luminance incident on it, as a sensor. This principle and the power function developed were used to model $V_{L Q D}$; which reveals that $V_{L Q D}$ in liters is a function of $E_{L U X}$ in Volts. The model of $V_{L Q D}$ was simulated for validation in Proteus 8.9 professional environment. The result obtained revealed that the model correctly indicated the height and volume of the liquid in the closed container

\section{REFERENCES}

[1]. Marick, S., Bera, S.K., and Bera, S.C. 2014. A Float Type Liquid Level Measuring System Using A Modified Inductive Transducer. Sens. Transducers. 182(11): 111-118.

[2] Wilner, L.B. 2007. Variable Capacitance Liquid Level Sensors. Rev. Sci. Instrum. 31(5): 501-507. https://doi.org/10.1063/1.1931234

[3] Bera, S.C., Mandal, H., Saha, S., and Dutta, A.A. 2014. Study of A Modified Capacitance-Type Level Transducer for Any Type of Liquid. IEEE Transactions on Instrumentation and Measurement. 46(2): 641-649. doi:10.1109/TIM.2013.2282194

[4] Toth, E.N., Meijer, G.C.M., and Van der Lee, C.M. 1997. A Planar Capacitive Precision Gauge for Liquid Level and Leakage Detection. IEEE Transactions on Instrumentation and Measurement. 46(2): 644-646. doi:10.1109/19.572052 
[5] Thathachary, S.V., George, B., and Kumar, V.J. 2013. A Resistive Potentiometer Type Transducer with Contactless Slide. Proceedings of Seventh International Conference on Sensing Technology (ICST). 501505. doi:10.1109/ICSensT.2013.6727703

[6] Pop, S., Bande, V., Pitika, D., and Ciascai, I. 2012. Dynamic measurement circuit for the resistive transducers. IEEE 18th International Symposium for Design and Technology in Electronic Packaging (SIITME). 259-262. doi:10.1109/SIITME.2012.6384387

[7] Yao, X., Zhang, Y., Gao, L., Yin., W., Yang, J., and Wang, C. 2009. Ground Water Level Measurements Using A Segmented Resistance Sensor. Proceedings of IEEE Instrumentation and Measurement Technology Conference. 1357-1360. doi:10.1109/IMTC.2009.5168667.

[8] Terzic J., Ngarajah C.R., and Alagir, M., 2010. Fluid Level Measurement In Dynamic Environments Using a Single Ultrasonic Sensor And Support Sensor Machined (SVM). Sensors and Actuators A: Physical. 161(11): 278-287. https://doi.org/10.1016/j.sna.2010.05.005

[9] Zhang., B., Wei, Y.J., Liu., W.Y., Zhang, Y.J., Yao, Z., Zhang, I., Yao, Z., Zhao, I.H., and Xiong, J.J. 2017. A Liquid Level Measurement Technique outside a Sealed Metal Container Based On Ultrasonic Impedance and Echo Energy. Sensors, 17(1): 185. https://doi.org/10.3390/s17010185

[10] Zhang., B., Wei, Y.J., Liu., W.Y., Zhang, Y.J., Yao, Z., Zhang, I., Yao, Z., Zhao, I.H., and Xiong, J.J. 2017. A Novel Ultrasonic Method for Liquid Level Measurement Based on the Balance of Echo Energy. Sensors, 17(4): 706. https://doi.org/10.3390/s17040706

[11] Saxena, T., Rumyantsev, S.L., Dutta P.S., and Shur, M. 2014. CdS Based Novel Photo-Impedance Light Sensor. Semicond. Sci. Technol. 29, 025002. doi:10.1088/0268-1242/29/2/025002

[12] Marinho, F., Carvalho, C.M., Apolinario, T.R., and L. Paulucci, L. 2019. Measuring Light with Light Dependent Resistors: An Easy Approach for Optics Experiments. European Journal of Physics, 40(3): 035801 .

[13] Abramson, J. 2017. Pre-calculus. Published by OpenStax, Rice University, Houston, Texas

[14] William J Palm, W.J. 2005. Introduction to MATLAB 7 for engineers, 2nd Edition. Published by McGraw.

[15] Salawu, R.I., Okafor, F.N., and Adetona, S.O. 2009. Evaluation of electrical load growth rate in a wholesale electricity market. 2nd International Conference on Adaptive Science \& Technology (ICAST), Accra, Ghana, 182-186. doi:10.1109/ICASTECH.2009.5409728.

[16] Labcenter Electronics Ltd 2019. Proteus Design Suite 8.9

[17] Singh, R., Gehlot, A., Singh, B., and Choudhury S. 2018. Arduino-Based Embedded Systems Interfacing. Simulation and LabVIEW GUI. CRC Press Taylor \& Francis Group

[18] Schmidt M. 2015. Arduino: A Quick-Start Guide, Second Edition. The Pragmatic Programmers, LLC

[19] Bayle, J. 2013. C Programming for Arduino, Published by Packt Publishing Ltd, Birmingham, UK

[20] https://www.arduino.cc/reference/en/language/functions/math/pow/ 